\title{
Prognostic nutritional index serves as a predictive marker of survival and associates with systemic inflammatory response in metastatic intrahepatic cholangiocarcinoma
}

\author{
This article was published in the following Dove Press journal: \\ OncoTargets and Therapy \\ 19 October 2016 \\ Number of times this article has been viewed
}

\author{
Chenyue Zhang ${ }^{1,2}$ \\ Haiyong Wang ${ }^{1,3}$ \\ Zhouyu Ning ${ }^{1,2}$ \\ Litao $\mathrm{Xu}^{1,2}$ \\ Liping Zhuang ${ }^{1,2}$ \\ Peng Wang ${ }^{1,2}$ \\ Zhiqiang Mengl,2 \\ 'Department of Integrative Oncology, \\ Fudan University Shanghai Cancer \\ Center, ${ }^{2}$ Department of Oncology, \\ Shanghai Medical College, Fudan \\ University, Shanghai, ${ }^{3}$ Department of \\ Radiation Oncology, Shandong Cancer \\ Hospital and Institute, Jinan, People's \\ Republic of China
}

Correspondence: Zhiqiang Meng; Peng Wang

Department of Integrative Oncology, Fudan University Shanghai Cancer

Center, 270 Dong An Road, Shanghai 200032, People's Republic of China

Tel +86 2I 64175590

Fax +86 2I 64437657

Email mengzhq@yeah.net;

wangp4I3@I63.com
Objective: The significance of the prognostic nutritional index (PNI) has been widely reported and confirmed in many types of cancers. However, few studies are available indicating its prognostic power in patients with intrahepatic cholangiocarcinoma (ICC). Thus, we investigated its relationship with overall survival (OS) to evaluate its role in predicting survival in patients with ICC.

Patients and methods: Between October 2011 and October 2015, 173 consecutive patients with pathologically confirmed locally advanced or metastatic ICC were enrolled. First, the correlations between PNI and clinical factors were analyzed among these patients. Next, univariate and multivariate analyses were conducted to evaluate the association between PNI and OS among these patients with ICC. In addition, the relationships between PNI and three typical systemic inflammatory response (SIR) markers - the neutrophil/lymphocyte ratio (NLR), the platelet/ lymphocyte ratio (PLR), and the lymphocyte/monocyte ratio (LMR) - were also assessed.

Results: A lower PNI was linked with a shorter OS in patients with ICC, as reflected obviously in the Kaplan-Meier analyses. The patients with ICC were divided into the locally advanced group and the metastatic group. Further analyses revealed that PNI is not associated with OS in the locally advanced group. However, in the subgroup of patients with metastatic ICC, a lower PNI significantly correlated with a worsened OS. The OS for patients with a low PNI is 5 months, whereas the OS is 10.17 months for patients with a high PNI. Multivariate analyses revealed that PNI is independently correlated with OS. We finally proved that PNI is negatively proportional to NLR and PLR and positively proportional to LMR.

Conclusion: Our results demonstrate that decreased PNI signifies a poor OS and is associated with SIR in patients with metastatic ICC. Therefore, it may serve as a valuable predictive marker in patients with metastatic ICC.

Keywords: prognostic nutritional index, SIR marker, predictive marker, metastatic, intrahepatic cholangiocarcinoma

\section{Introduction}

Intrahepatic cholangiocarcinoma (ICC), one of the most devastating malignancies, constitutes $15 \%$ of all primary liver cancers. ${ }^{1,2}$ ICC is highly dangerous and signifies a dismal prognosis. Despite being a rare malignancy, the incidence and mortality of ICC are increasing at an unprecedented rate. ${ }^{3-6}$ Unlike other tumors originating from the liver and gastrointestinal tract, the pathogenesis of ICC remains complex and obscure, thus making the disease more intractable and challenging. ${ }^{7}$ Moreover, patients with 
ICC have a median survival of not $>12$ months, even after different treatments such as surgery, systemic chemotherapy, and radiotherapy. ${ }^{8}$ However, clinical data have shown that survival rates vary significantly among patients with ICC. The commonly acknowledged factors that may serve as predictors for survival include intrahepatic satellite lesions, lymph node invasion, and distant metastasis. ${ }^{9-11}$

Cancer is universally acknowledged as a malnourishing disease. ${ }^{12,13}$ Patients with such malignancy can suffer from drastic weight loss and nutritional deficiency, which can greatly contribute to the mortality and morbidity. ${ }^{14-16}$ Therefore, an impaired nutritional status may have an adverse impact on the overall survival (OS) of cancer patients. A deteriorated nutritional status is intimately associated with delayed wound healing and weakening of the immune system, which can be reflected by the reduction in the amounts of neutrophils, macrophages, and lymphocytes. ${ }^{17}$ Besides, many studies have demonstrated that patients subject to malnutrition are more likely to develop postoperative complications due to the disorder of the immune functions. ${ }^{18,19}$

Previous studies have reported that the nutritional status can be assessed by a number of biochemical indices, such as body mass index, prognostic nutritional index (PNI), nutrition risk index, and nutritional risk score. ${ }^{20,21}$ Among all these prognostic indexes, PNI has been recognized as an accurate parameter reflecting patients' nutritional status. It is determined by the serum albumin levels and the lymphocyte count in the peripheral blood and has been demonstrated to be valuable in predicting the outcome of patients with malignant tumors originating from the digestive system. ${ }^{22,23}$

Until now, the exact mechanisms of reduced PNI remain to be explored, which has been recognized as a manifestation of multifactorial effects. A multitude of studies have shown that a systemic inflammatory response (SIR) may be involved in the cancer-related malnutrition, thus leading to a worsened prognosis among cancer patients. ${ }^{24,25}$ Systemic inflammation has shown to be related to unfavorable prognosis, possibly by affecting tumor growth, angiogenesis, metastasis as well as their responses to treatments via modulating complex metabolic processes. The most recognized and widely used SIR markers involved the neutrophil/lymphocyte ratio (NLR), the platelet/lymphocyte ratio (PLR), and the lymphocyte/ monocyte ratio (LMR), and it remains to be determined whether there are any associations between PNI and SIR markers in patients with ICC.

Therefore, the purpose of the present study is to determine the role of PNI in patients with ICC and its association with SIR markers.

\section{Patients and methods}

\section{Patients}

From October 2011 to October 2015, a total of 173 patients (107 [61.8\%] males and 66 [38.2\%] females), who were pathologically diagnosed with ICC at the Department of Integrative Oncology, Fudan University Shanghai Cancer Center, Shanghai, People's Republic of China, were enrolled in the present study. These patients were subject to the standard radiological procedures, such as contrast-enhanced abdominal computed tomography scans and magnetic resonance imaging, which have also confirmed their diagnoses. The criteria for locally advanced or metastatic stage were based on the guidelines of the American Joint Committee on Cancer (AJCC) staging. Only 36 patients had a diagnosis of locally advanced cancer among all the patients, accounting for a relatively smaller proportion $(20.8 \%)$, whereas the remaining patients were at the metastatic stage. The number of patients who had CA199 of $\geq 1,000 \mathrm{IU} / \mathrm{mL}$ summed up to 64 , accounting for $37.0 \%$ of all the patients. Among all the patients, 99 patients received gemcitabine-based chemotherapy, while the remaining 74 patients were subjected to 5-fluorouracil-based chemotherapy. Table 1 summarizes the characteristics of all the enrolled patients. This research was approved by the ethics committee of Fudan University Shanghai Cancer Center, Shanghai, People's Republic of China, and written informed consent was obtained from all the enrolled patients.

Table I The association between PNI and clinical factors in intrahepatic cholangiocarcinoma

\begin{tabular}{|c|c|c|c|}
\hline Variables & $\begin{array}{l}\mathrm{PNI}<47 . \mathrm{I} \\
(\mathrm{n}=86)\end{array}$ & $\begin{array}{l}P N I \geq 47 . I \\
(n=87)\end{array}$ & $P$-value \\
\hline Age (years), mean \pm SD & $60.29 \pm 11.85$ & $57.39 \pm 10.62$ & 0.549 \\
\hline \multicolumn{4}{|l|}{ Gender } \\
\hline Male & 57 & 50 & \multirow[t]{2}{*}{0.233} \\
\hline Female & 29 & 37 & \\
\hline \multicolumn{4}{|l|}{ Stage } \\
\hline Locally advanced & 18 & 18 & \multirow[t]{2}{*}{0.969} \\
\hline Metastatic & 68 & 69 & \\
\hline \multicolumn{4}{|l|}{ CAI99 } \\
\hline$<\mathrm{I}, 000 \mathrm{IU} / \mathrm{mL}$ & 48 & 61 & \multirow[t]{2}{*}{0.051} \\
\hline$\geq \mathrm{I}, 000 \mathrm{IU} / \mathrm{mL}$ & 38 & 26 & \\
\hline \multicolumn{4}{|l|}{ NLR } \\
\hline$<3.21$ & 30 & 56 & \multirow[t]{2}{*}{$<0.000$ I } \\
\hline$\geq 3.21$ & 56 & 31 & \\
\hline \multicolumn{4}{|l|}{ PLR } \\
\hline$<138$ & 30 & 58 & \multirow[t]{2}{*}{$<0.000$ I } \\
\hline$\geq 138$ & 56 & 29 & \\
\hline \multicolumn{4}{|l|}{ LMR } \\
\hline$<3$ & 57 & 27 & \multirow[t]{2}{*}{$<0.0001$} \\
\hline$\geq 3$ & 29 & 60 & \\
\hline
\end{tabular}

Abbreviations: PNI, prognostic nutritional index; NLR, neutrophil/lymphocyte ratio; PLR, platelet/lymphocyte ratio; LMR, lymphocyte/monocyte ratio; SD, standard deviation. 


\section{Calculation of SIR markers and PNI}

Blood samples were taken for neutrophil, monocyte, platelet, and lymphocyte counts. The levels of serum albumin were also measured. All these tests were performed prior to all the treatments. NLR was derived by dividing the absolute neutrophil count by the absolute lymphocyte count. Likewise, PLR was derived by dividing the absolute platelet count by the absolute lymphocyte count. LMR was derived by dividing the absolute lymphocyte count by the absolute monocyte count. The median NLR, PLR, and LMR were 3.21, 138, and 3, respectively, and were chosen as the cutoffs. The PNI was calculated according to the acknowledged formula: $10 \times$ serum albumin $(\mathrm{g} / \mathrm{dL})+0.5 \% \times$ total lymphocyte number $\left(\right.$ per $\mathrm{mm}^{3}$ ). Provided that 47.1 was the median value among the 173 patients, we therefore classified PNI scores into two groups: $<47.1$ and $\geq 47.1$ for subsequent studies.

\section{Statistical analyses}

Values were expressed as mean \pm standard deviation. Statistical comparisons were done using chi-square tests. Cumulative survival curves of patients in different groups were depicted by Kaplan-Meier analysis, and the log-rank test was adopted to estimate the difference in survival. The associations between several variables with survival were first evaluated by univariate analyses. Multivariate analysis was then conducted using the Cox regression model adjusted for cancer stages and CA199. A $P$-value of $>0.05$ was considered to be statistically significant. All these tests were performed using SPSS 17.0 software (SPSS Inc., Chicago, IL, USA).

\section{Results}

\section{Patient characteristics and clinical features}

In total, 173 patients (107 [61.8\%] males and 66 [38.2\%] females) were enrolled in this study. According to the AJCC staging, a total of $36(20.8 \%)$ patients were diagnosed with locally advanced ICC, while the other 137 patients were diagnosed with metastatic ICC. All the173 patients with ICC received standard chemotherapy, among whom 99 patients received gemcitabine-based chemotherapy and 74 patients were subjected to 5-fluorouracil-based chemotherapy. Details of the clinicopathological characteristics are given in Table 1.

\section{Decreased PNI signifies shorter OS in patients with metastatic ICC}

In order to explore the role of PNI in patients with ICC, we evaluated the relationship between PNI and OS among these patients with ICC. A lower PNI was found to be obviously related to a shorter OS in patients with ICC, as shown in the Kaplan-Meier analyses. Patients with a low PNI has an unsatisfactory OS of 7.2 months, whereas patients with a high PNI has an improved OS of 12.9 months (log-rank $=7.673$, $P=0.0056$; Figure 1A). Besides, the role of PNI was evaluated in both the locally advanced and the metastatic ICC, respectively. We have shown that there is a lack of accurate correlation between a low PNI and a worsened OS in patients with locally advanced ICC (Figure 1B). However, in patients with metastatic ICC, we have seen a rather significant correlation between PNI and OS. Among the subgroup of metastatic ICC, patients with a low PNI only had a median survival of 5.00 months, whereas the median survival of patients with a relatively higher PNI was 10.17 months, twice as that of the patients with lower PNI (Figure 1C). Therefore, we have drawn the conclusion that decreased PNI signifies shorter OS in patients with metastatic ICC.

\section{$\mathrm{PNI}$ can predict for OS in patients with ICC}

To determine the prognostic power of PNI for OS in patients with ICC, we first performed univariate analyses. Results have shown that among all the variables, cancer stage, CA199, and PNI have an enormous impact on OS. In order to verify the independent impact of PNI on OS, we performed multivariate analyses to counteract the influence of cancer stage and CA199 on OS. Multivariate analysis confirmed that PNI served as an independent prognostic factor for OS in patients with ICC after adjusting for confounding factors such as tumor stage and CA199. The hazard ratio of PNI (low vs high) was 0.646 , with $95 \%$ confidence interval: $0.458-0.911$. Relevant data are collected in Table 2.

\section{$\mathrm{PNI}$ can reflect the SIR in patients with ICC}

Since some studies have reported that the deteriorated nutritional status may be mediated by the augmented SIR, we then tried to explore whether there are any close links between PNI and NLR, PLR, and LMR, the three typical SIR markers in the patients with ICC. In the cohort of patients with ICC, there was a consistent trend between being in a malnutritional state and a boost in SIR, which can be reflected by the elevated NLR, PLR, and the reduced LMR. The associations between PNI and some clinical factors including SIR markers in patients with ICC are listed in Table 1. Besides, among the 173 patients with ICC, PNI was found to be in positive proportion to LMR as shown in Figure $2 \mathrm{C}(r=0.528, P<0.0001)$, 

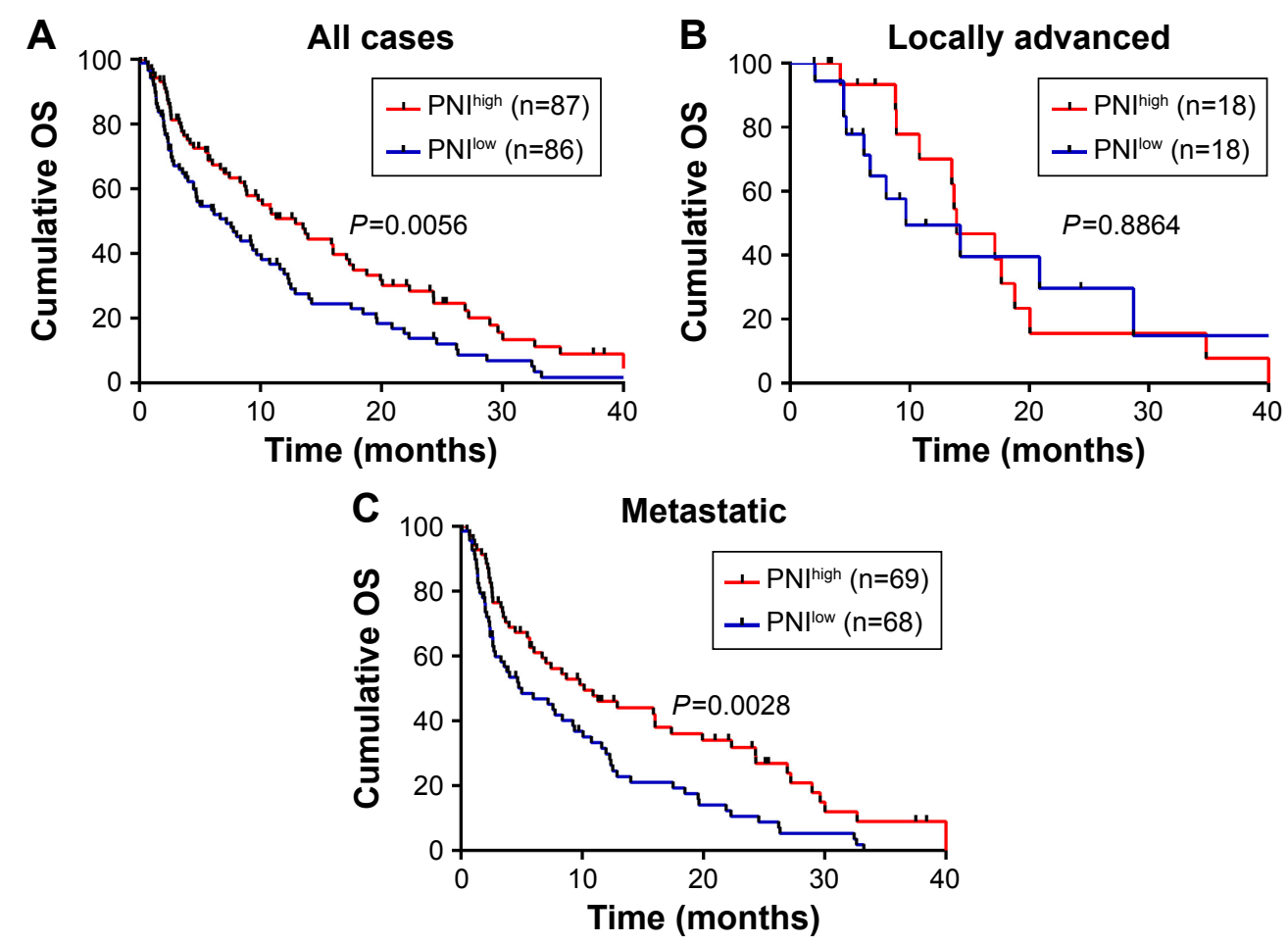

Figure I PNI was associated with OS in patients with metastatic ICC.

Notes: Kaplan-Meier curves for OS in the patients with ICC: all cases (A), locally advanced (B), and metastatic (C). The median PNI was chosen as the cutoff between the low and the high PNI.

Abbreviations: OS, overall survival; ICC, intrahepatic cholangiocarcinoma; PNI, prognostic nutritional index.

but its elevation was in line with the reduction in NLR and PLR as shown in Figure 2A and B $(r=-0.404, P<0.0001$ and $r=-0.402, P<0.0001$, respectively). Since we divided the patients with ICC into the locally advanced and metastatic groups, we then analyzed the relationship between PNI and the SIR markers in these subgroup patients. Our results confirmed PNI's positive correlation between LMR $(r=0.578, P<0.0001)$, and its negative correlation with NLR and PLR $(r=-0.465, P<0.0001$ and $r=-0.439, P<0.0001$, respectively; Figure 2) in patients with metastatic ICC. And in patients with locally advanced ICC, PNI also correlated positively with LMR and negatively with NLR and PLR, despite the association not being statistically significant. Therefore, we demonstrated that decreased PNI may signify an enhanced SIR in patients with metastatic ICC.

\section{Discussion}

In the present study, we aimed to ascertain the role of PNI in patients with ICC. We found that a low PNI was significantly correlated with a shorter OS in patients with ICC. Furthermore, we also divided the patients with ICC into the locally advanced group and the metastatic group. Deeper analyses

Table 2 Univariate and multivariate Cox regression analyses of PNI and OS in patients with intrahepatic cholangiocarcinoma $(\mathrm{N}=173)$

\begin{tabular}{|c|c|c|c|}
\hline Variables & Case number & Hazard ratio $(95 \% \mathrm{Cl})$ & $P$-value \\
\hline \multicolumn{4}{|l|}{ Univariate analysis } \\
\hline Age ( $<60$ years vs $\geq 60$ years) & $79 / 94$ & $0.85 I(0.607-1.193)$ & 0.349 \\
\hline Gender (male vs female) & $107 / 66$ & I.I4I (0.809-I.625) & $0.44 I$ \\
\hline Cancer stage (locally advanced vs metastatic) & $36 / 137$ & $1.668(1.063-2.617)$ & 0.026 \\
\hline CAI $99(<I, 000 \mathrm{IU} / \mathrm{mL}$ vs $\geq \mathrm{I}, 000 \mathrm{IU} / \mathrm{mL})$ & $109 / 64$ & $2.273(1.584-3.264)$ & $<0.0001$ \\
\hline PNI (low vs high) & $86 / 87$ & $0.62(0.442-0.873)$ & 0.006 \\
\hline \multicolumn{4}{|l|}{ Multivariate analysis } \\
\hline Cancer stage (locally advanced vs metastatic) & $36 / 137$ & $1.640(1.043-2.579)$ & 0.032 \\
\hline CAI99 (<I,000 IU/mL vs $\geq I, 000 \mathrm{IU} / \mathrm{mL})$ & $109 / 64$ & $2.122(1.475-3.05 I)$ & $<0.0001$ \\
\hline PNI (low vs high) & $86 / 87$ & $0.646(0.458-0.911)$ & 0.013 \\
\hline
\end{tabular}

Abbreviations: PNI, prognostic nutritional index; OS, overall survival; $\mathrm{Cl}$, confidence interval. 
A

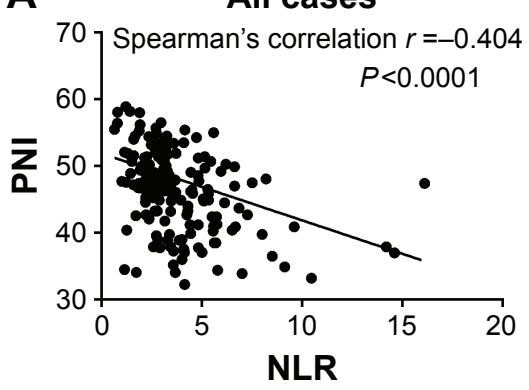

B

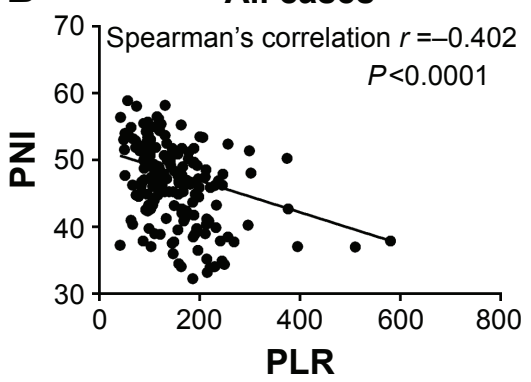

C

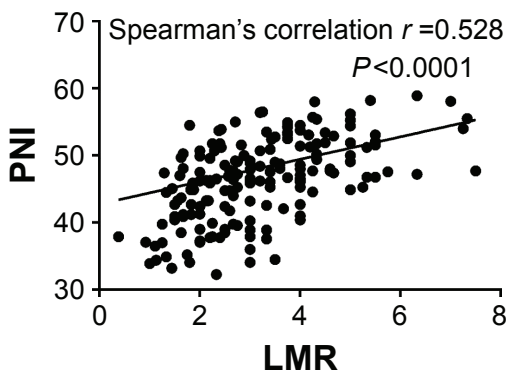

Locally advanced
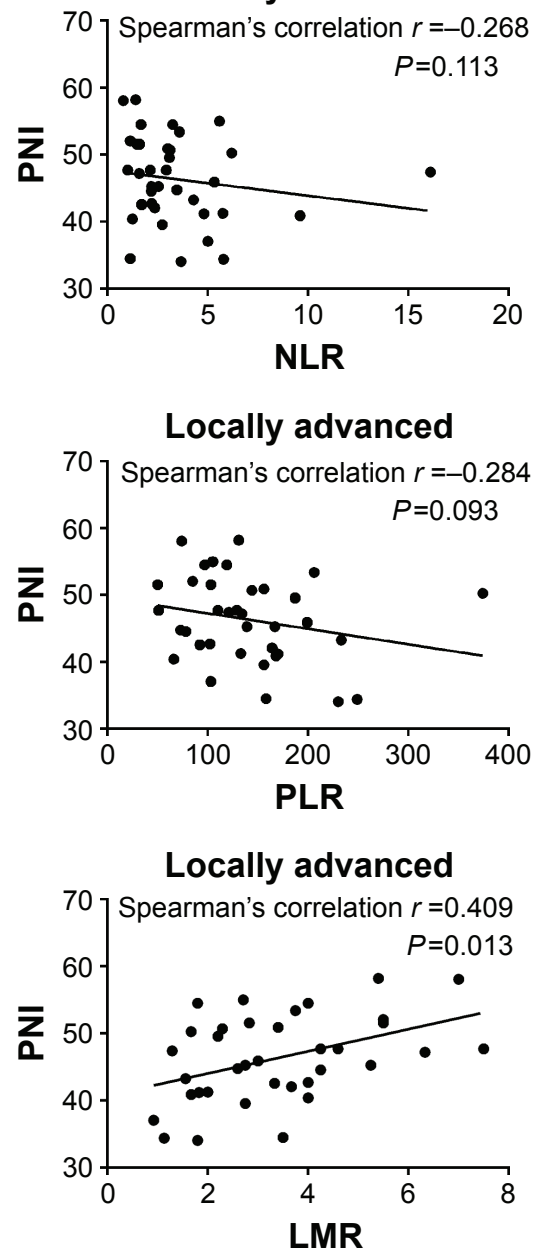

Metastatic

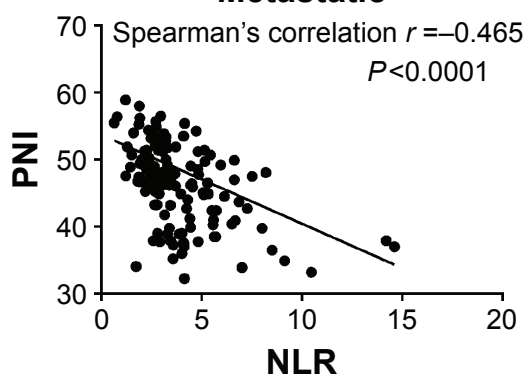

Metastatic

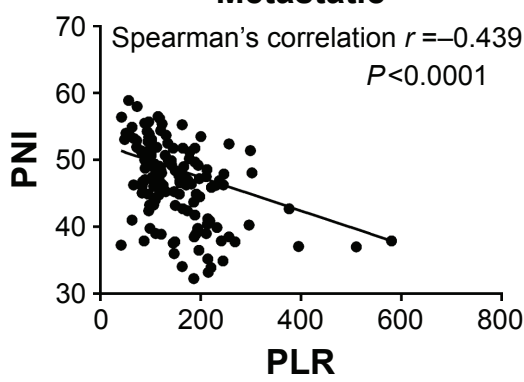

Metastatic

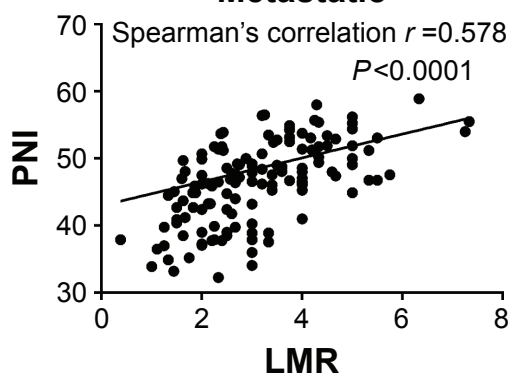

Figure $2 \mathrm{PNI}$ was associated with the systemic inflammatory response markers.

Notes: The association between PNI and NLR (A), PLR (B), and LMR (C) was evaluated using Spearman's association. The correlation was evaluated in all the cases, and in the subgroups of the locally advanced and patients with metastatic ICC.

Abbreviations: PNI, prognostic nutritional index; NLR, neutrophil/lymphocyte ratio; PLR, platelet/lymphocyte ratio; LMR, lymphocyte/monocyte ratio; ICC, intrahepatic cholangiocarcinoma.

have revealed that a lower PNI signifies a shorter OS in the metastatic group. Therefore, it can be served as a valuable prognostic predictor for OS in patients with ICC, especially in those with metastatic ICC. In addition, we have found that to some point PNI can reflect the SIR in patients with ICC, as shown in the close link between PNI and NLR, PLR, and LMR.

Cancer patients are often subject to malnutrition due to the adverse effects imposed by the tumors on the digestive system. ${ }^{26}$ Patients with ICC often suffer from digestive problems such as nausea and vomiting, and most of them undergo operation and chemotherapy, which may exacerbate the unfavorable digestive symptoms, thus aggravating their malnutritional status. Previous studies have demonstrated that the anorexia may affect the life quality and prognosis in cancer patients. ${ }^{27,28}$ However, the explanations of tumor-induced malnutrition are complex and the process may involve the delicate interplay between the tumor and the host. ${ }^{29,30}$ Thus, determination of patients who are likely to become malnutritional and have unfavorable prognosis has always been a challenging subject of studies.

Indeed, the PNI was first introduced by Onodera et $\mathrm{al}^{31}$ to evaluate patients with gastric cancers undergoing surgeries with regard to their catabolic state. Thereafter, researchers have investigated its functions in other types of cancers, such as colorectal, esophageal, and hepatocellular carcinomas. Studies have shown that low PNI correlates with the shorter OS in various types of cancers. ${ }^{32-34}$ In the present study, we have shown that decreased PNI signifies shorter OS in patients with ICC. We then further found that in patients with the metastatic ICC, there is a more intimate and evident correlation between PNI and OS. As reflected from the definition 
of PNI, we can easily learn that PNI was determined by two factors, the levels of serum albumin and the lymphocyte count in the peripheral blood. Our results are in line with a series of previous studies reporting the adverse effects of reduced serum albumin and lymphocyte counts in some types of cancers. ${ }^{35,36}$ Interestingly, we have found that PNI is closely correlated with OS in patients with metastatic ICC. However, this association was not found in patients with locally advanced ICC. This could be attributed to the disparate characteristics between the two respective cohorts of patients. Patients with metastatic ICC often suffer more severely from weight and muscle loss, which is not only induced by less food intake but also triggered by the adverse consequence of interaction between tumor and the host. Such interactions in these patients are mediated by a sophisticated network of cytokines and tumor-derived factors. These cytokines could in turn induce cancer anorexia-cachexia syndrome in these patients with ICC. ${ }^{24-26}$ Metastatic patients with ICC are often subject to much more tumor burden. Therefore, their tumors would elicit abundant inflammatory cytokines, which would aggravate their inflammatory status, resulting in a worsened OS. In our study, PNI reflected the status of systemic inflammation of the patients with ICC and was associated with OS, which implied that PNI can be reflective of tumor burden. Thus, it is quite understandable that the association of PNI with OS was found in patients with metastatic ICC not locally advanced.

PNI is indeed one of the systemic inflammatory markers, and it is reflective of both the nutritional and immunologic status. Albumin is commonly tested in clinical applications, indicating the nutritional status. Decreased albumin expression has been considered an indication of malnutrition and weak immune defense system, and its drastic reduction often gives a hint of postoperative complications to clinicians. In addition, one study has emphasized the contributing role of the inflammatory response in the albumin reduction in many cancer patients. ${ }^{37}$

Inflammation has been recognized as a central feature of cancer and serves as a contributing culprit in accelerating cancer via promoting tumor proliferation, angiogenesis, and metastasis. ${ }^{6}$ Systemic inflammation has been considered valuable reflecting poor prognosis in cancers. Therefore, the inflammatory markers have been widely investigated in the cancer field. Rapid cancer progression and poor prognoses were reported to be concomitant with some inflammatory markers, as exemplified by NLR, PLR, and LMR. ${ }^{38,39}$ Considering that PNI is also indicative of the inflammatory status, we therefore verified whether there are any close links with PNI and the SIR markers in these inpatients with ICC, especially those with metastatic ICC. We have found the positive relationship between PNI and LMR, and the negative relationship between NLR and PLR in patients with ICC, specifically in those in the metastatic stage.

Taken together, we found that PNI can be served as a valuable marker to predict prognosis in the patients with metastatic ICC. We also demonstrated some relevance between PNI and the SIR in patients with ICC. As described, PNI could serve as a marker indicative of the complex interplay between tumor and the host. Besides, PNI can be easily calculated from the routine blood test. Therefore, PNI is a favorable indication more reflective of the overall status of the cancer patients. Thus, it can be used to assess the prognosis of cancer patients.

Therefore, the assessment of PNI is highly desired in clinical application. As to patients with decreased PNI, some alternative therapeutic methods and more frequent follow-up can be provided, which may prolong their survival.

However, this study also has some limitations. First, some other parameters involving in inflammation such as CRP, TNF- $\alpha$, and interleukins were not measured in our study because they are not routinely tested in clinical applications. Second, being a retrospective research, it has its own flaws since it was conducted at a single medical center with a relatively smaller number of cases. Thus, more studies are warranted to further validate the role of PNI and the possible mechanism in determining the prognosis of ICC.

\section{Acknowledgment}

This study was supported by the Natural Science Foundation of China (number 81273954).

\section{Disclosure}

The authors report no conflicts of interest in this work.

\section{References}

1. Patel T. Cholangiocarcinoma - controversies and challenges. Nat Rev Gastroenterol Hepatol. 2011;8(4):189-200.

2. Patel T. Worldwide trends in mortality from biliary tract malignancies. BMC Cancer. 2002;2:10.

3. Braconi C, Patel T. Cholangiocarcinoma: new insights into disease pathogenesis and biology. Infect Dis Clin North Am. 2010;24(4):871-884.

4. Chu KM, Lai EC, Al-Hadeedi S, et al. Intrahepatic cholangiocarcinoma. World J Surg. 1997;21(3):301-305; discussion 305-306.

5. Kawarada Y, Yamagiwa K, Das BC. Analysis of the relationships between clinicopathologic factors and survival time in intrahepatic cholangiocarcinoma. Am J Surg. 2002;183(6):679-685.

6. Hanahan D, Weinberg RA. Hallmarks of cancer: the next generation. Cell. 2011;144(5):646-674.

7. Gunter MJ, Stolzenberg-Solomon R, Cross AJ, et al. A prospective study of serum C-reactive protein and colorectal cancer risk in men. Cancer Res. 2006;66(4):2483-2487. 
8. Jaiswal M, LaRusso NF, Burgart LJ, Gores GJ. Inflammatory cytokines induce DNA damage and inhibit DNA repair in cholangiocarcinoma cells by a nitric oxide-dependent mechanism. Cancer Res. 2000;60(1): 184-190.

9. Jackson JR, Seed MP, Kircher CH, Willoughby DA, Winkler JD. The codependence of angiogenesis and chronic inflammation. FASEB $J$. 1997;11(6):457-465.

10. Gabay C, Kushner I. Acute-phase proteins and other systemic responses to inflammation. N Engl J Med. 1999;340(6):448-454.

11. Krenn-Pilko S, Langsenlehner U, Thurner EM, et al. The elevated preoperative platelet-to-lymphocyte ratio predicts poor prognosis in breast cancer patients. Br J Cancer. 2014;110(10):2524-2530.

12. Theologides A. Pathogenesis of cachexia in cancer. A review and a hypothesis. Cancer. 1972;29(2):484-488.

13. Costa G. Cachexia, the metabolic component of neoplastic diseases. Progr Exp Tumor Res. 1963;3(7 Pt 2):321.

14. DeWys WD, Begg C, Lavin PT, et al. Prognostic effect of weight loss prior to chemotherapy in cancer patients. Eastern Cooperative Oncology Group. Am J Med. 1980;69(4):491-497.

15. Andreyev HJN, Norman AR, Oates J, Cunningham D. Why do patients with weight loss have a worse outcome when undergoing chemotherapy for gastrointestinal malignancies? Eur J Cancer. 1998; 34(4):503-509.

16. Conti S, West JP, Fitzpatrick HF. Mortality and morbility after esophagogastrectomy for cancer of the esophagus and cardia. Am Surg. 1977; 43(2):92.

17. Schneider SM, Veyres $P$, Pivot $X$, et al. Malnutrition is an independent factor associated with nosocomial infections. Br J Nutr. 2004;92(1): 105-111.

18. Windsor JA, Hill GL. Risk factors for postoperative pneumonia. The importance of protein depletion. Ann Surg. 1988;208(2):209-214.

19. Bozzetti F, Gavazzi C, Miceli R, et al. Perioperative total parenteral nutrition in malnourished, gastrointestinal cancer patients: a randomized, clinical trial. JPEN J Parenter Enteral Nutr. 2000;24(7):14.

20. Smale BF, Mullen JL, Buzby GP, Rosato EF. The efficacy of nutritional assessment and support in cancer surgery. Cancer. 1981;47(10): 2375-2381.

21. Hendifar A, Osipov A, Khanuja J, et al. Influence of body mass index and albumin on perioperative morbidity and clinical outcomes in resected pancreatic adenocarcinoma. PLoS One. 2016;11(3):e0152172.

22. Ishizuka M, Oyama Y, Abe A, Tago K, Tanaka G, Kubota K. Prognostic nutritional index is associated with survival after total gastrectomy for patients with gastric cancer. Anticancer Res. 2014;34(8):4223-4229.

23. Ikeguchi M, Urushibara S, Shimoda R, Yamamoto M, Maeta Y, Ashida K. Inflammation-based prognostic scores and nutritional prognostic index in patients with locally-advanced unresectable colorectal cancer. World J Surg Oncol. 2014;12:210.

24. Fearon KC, Voss AC, Hustead DS; Cancer Cachexia Study Group. Definition of cancer cachexia: effect of weight loss, reduced food intake, and systemic inflammation on functional status and prognosis. Am J Clin Nutr. 2006;83(6):1345-1350.
25. Deans DA, Tan BH, Wigmore SJ, et al. The influence of systemic inflammation, dietary intake and stage of disease on rate of weight loss in patients with gastro-oesophageal cancer. Br J Cancer. 2009;100(1):63-69.

26. Richter E, Denecke A, Klapdor S, Klapdor R. Parenteral nutrition support for patients with pancreatic cancer-improvement of the nutritional status and the therapeutic outcome. Anticancer Res. 2012;32(5): 2111-2118.

27. Barbosa-Silva MC, Barros AJ. Bioelectric impedance and individual characteristics as prognostic factors for post-operative complications. Clin Nutr. 2005;24(5):830-838.

28. Kuzu MA, Terzioğlu H, Genç V, et al. Preoperative nutritional risk assessment in predicting postoperative outcome in patients undergoing major surgery. World J Surg. 2006;30(3):378-390.

29. Argiles JM, Moore-Carrasco R, Fuster G, Busquets S, Lopez-Soriano FJ. Cancer cachexia: the molecular mechanisms. Int J Biochem Cell Biol. 2003;35(4):405-409.

30. Laviano A, Meguid MM, Rossi-Fanelli F. Cancer anorexia: clinical implications, pathogenesis, and therapeutic strategies. Lancet Oncol. 2003;4(11):686-694.

31. Onodera T, Goseki N, Kosaki G. Prognostic nutritional index in gastrointestinal surgery of malnourished cancer patients. Nihon Geka Gakkai Zasshi. 1984;85(9):1001-1005.

32. Pinato DJ, North BV, Sharma R. A novel, externally validated inflammation-based prognostic algorithm in hepatocellular carcinoma: the prognostic nutritional index (PNI). Br J Cancer. 2012;106(8):1439-1445.

33. Nozoe T, Kohno M, Iguchi T, et al. The prognostic nutritional index can be a prognostic indicator in colorectal carcinoma. Surg Today. 2012; 42(6):532-535.

34. Jiang N, Deng JY, Ding XW, et al. Prognostic nutritional index predicts postoperative complications and long-term outcomes of gastric cancer. World J Gastroenterol. 2014;20(30):10537-10544.

35. Nozoe T, Kimura Y, Ishida M, Saeki H, Korenaga D, Sugimachi K. Correlation of pre-operative nutritional condition with post-operative complications in surgical treatment for oesophageal carcinoma. Eur $J$ Surg Oncol. 2002;28(4):396-400.

36. Kanda M, Fujii T, Kodera Y, Nagai S, Takeda S, Nakao A. Nutritional predictors of postoperative outcome in pancreatic cancer. Br J Surg. 2011;98(2):268-274.

37. Esper DH, Harb WA. The cancer cachexia syndrome: a review of metabolic and clinical manifestations. Nutr Clin Pract. 2005;20(4): 369-376.

38. Nishijima TF, Muss HB, Shachar SS, Tamura K, Takamatsu Y. Prognostic value of lymphocyte-to-monocyte ratio in patients with solid tumors: a systematic review and meta-analysis. Cancer Treat Rev. 2015;41(10):971-978.

39. Chen Q, Yang LX, Li XD, et al. The elevated preoperative neutrophil-to-lymphocyte ratio predicts poor prognosis in intrahepatic cholangiocarcinoma patients undergoing hepatectomy. Tumour Biol. 2015;36(7):5283-5289.
OncoTargets and Therapy

\section{Publish your work in this journal}

OncoTargets and Therapy is an international, peer-reviewed, open access journal focusing on the pathological basis of all cancers, potential targets for therapy and treatment protocols employed to improve the management of cancer patients. The journal also focuses on the impact of management programs and new therapeutic agents and protocols on

\section{Dovepress}

patient perspectives such as quality of life, adherence and satisfaction. The manuscript management system is completely online and includes a very quick and fair peer-review system, which is all easy to use. Visit http://www.dovepress.com/testimonials.php to read real quotes from published authors. 